\title{
POLÍTICAS DE PROTECCIÓN A LA INFANCIA Y ADOLESCENCIA EN URUGUAY. \\ UNA APROXIMACIÓN A LOS PROGRAMAS CON METODOLOGÍA DE PROXIMIDAD.
}

\begin{abstract}
CHILDREN AND ADOLESCENTS PROTECTION POLICIES IN URUGUAY. AN APPROACH TO PROGRAMS WITH PROXIMITY METHODOLOGY.
\end{abstract}

\author{
Victoria Lima Benitez ${ }^{1}$ \\ Investigadora independiente \\ Montevideo, Uruguay \\ https:/ / orcid.org/0000-0003-4029-4974 \\ victorialima1991@gmail.com
}

\section{Resumen}

El artículo se centra en el análisis de las políticas de protección a la infancia y adolescencia en Uruguay, específicamente en tres programas con metodología de proximidad; caracterizados por tener una modalidad de intervención focalizada, por la territorialización, la interinstitucionalidad y la cercanía, se analizarán las tensiones y los desafíos que tienen estos programas atendiendo a si generan transformaciones reales en las trayectorias de vida o si se trata de procesos de individualización de la pobreza. En materia infancia y adolescencia, Uruguay es uno de los Estados que ha ratificado la Convención Sobre los Derechos del Niño y a nivel nacional se enmarca en el Código de la Niñez y Adolescencia por lo que estas categorías deberían ser prioridad en la agenda política.

Ahora, ¿estos programas de protección a infancia y adolescencia protegen o acaban siendo una especie de tutelaje?

Palabras clave: Políticas públicas, Programas sociales, Estado, Infancia y Adolescencia.

\section{Abstract}

This article is focused on the analysis of the children and adolescents protection policies in Uruguay. Specifically, on three programs that are aimed at having a focused intervention mode by territorialization, inter-institutionalism and proximity. It will analyze the tensions and challenges of these programs, taking into account whether they generate real transformations in life trajectories or whether they are processes of individualization of poverty. Uruguay's State has ratified the Convention on the

1 Magister en Derecho de Familia e Infancia por la Universidad de Barcelona (UB). Licenciada en Trabajo Social por la Universidad de la República de Uruguay (UDELAR). 
rights of the children and at a nation level is framed under the code of childhood and adolescence, because of this reason the category should be a priority of the political agenda.

Considering this, do these children and adolescents protection policies protect them, or is this just a type of tutoring?

Keywords: Public policies, Social programs, State, Childhood and Adolescence.

\section{INTRODUCCIÓN}

Uruguay es un país que históricamente ha sido caracterizado por tener un gran componente de intervencionismo estatal en diferentes áreas. Una que resulta ser muy clara es la arena social y específicamente gran presencia en lo que refiere a la conducción de las políticas públicas en la cual los gobiernos son quienes las planifican y diseñan. Se representan y enmarcan bajo un sistema de protección social híbrido en el cual conviven políticas universales con políticas de corte focalizado (Alegre y Filgueira, 2009).

En los primeros años del siglo XXI en diferentes países de América Latina ocurre una especie de oleaje de gobiernos de izquierda. Esta situación no fue ajena a Uruguay cuando en el año 2005 llega por primera vez al gobierno el Frente Amplio, partido político que viene con una novedosa agenda de gobierno estableciendo un punto de inflexión en el tratamiento de la órbita pública. La matriz de protección social sufre grandes transformaciones, y el aparato estatal despliega una batería de dispositivos sociales en diferentes áreas y con diferentes poblaciones objetivos marcadas por tramos de edad con la premisa de disminuir la brecha social que atravesaba el país. Bajo esta coyuntura de transformación es que se crean programas sociales con metodologías de proximidad destinados al trabajo con la primera infancia, adolescentes y núcleos familiares en situación de vulnerabilidad.

En el presente documento se realizará una aproximación al análisis de la situación uruguaya en materia de intervención pública desde el año 2005 a la actualidad, y específicamente se intentará hacer un aporte en lo que refiere al estudio del alcance y los desafíos de los programas de cercanía. Previamente se deberá realizar un breve racconto histórico en materia de intervención pública en Uruguay lo cual permitirá al lector tener un mayor entendimiento de la situación local. Asimismo, se retomarán aportes actuales los cuales intentarán dar cuenta de la situación en la que se encuentra la política pública en lo que refiere a programas sociales destinados a: infancia, 
adolescencia y familia dejando abierta la discusión acerca de los logros y dificultades de los mismos.

En un primer momento se definirán las políticas públicas y sus características hasta llegar al concepto de política social universal y focalizada. Desde allí, se pasará a un breve recorrido histórico, desde mediados del siglo XX hasta la actualidad en Uruguay, presentando las peculiaridades de las políticas. Finalmente se presentarán tres de las específicas dirigidas a infancia y adolescencia conectándolo con el marco normativo tanto nacional como internacional que nos rige. Por último, se analizarán los desafíos y las tensiones actuales de programas sociales dirigidos a familia, infancia y adolescencia.

Desde el comienzo, es menester definir la categoría infancia, se partirá sosteniendo la multiplicidad de definiciones que tiene este concepto al ser amplio y tautológico "... no representa una única definición universal, sino que, por el contrario, se materializa en una multiplicidad de realidades" (Ravetllat,2015, p.1). La infancia como categoría social representa a individuos sujetos de derecho a los cuales se debe proteger "Hoy en día parece existir un acuerdo acerca de las características generales de la infancia y sobre la necesidad de protección de la que, en parte, deriva la discusión en relación con los derechos de los niños y su ejercicio" (González, 2011, p.17).

Para finalizar, se expondrán las conclusiones las cuales intentarán dejar abiertas ciertas discusiones que podrán ser retomadas en próximas publicaciones $\mathrm{y} / \mathrm{o}$ investigaciones.

En cuanto al diseño que tendrá el artículo, será de orden cualitativo y la técnica metodológica a utilizar será el análisis de datos secundarios, Respecto a esta técnica Kelder, (2005) señala que los datos cualitativos utilizando esta estrategia metodológica pueden ser analizados desde múltiples perspectivas, a su vez, permite la obtención de respuestas que van a ser diferentes a las que se obtuvieron a partir de los datos originales agregando confianza al momento de interpretar los datos.

\section{DEL CONCEPTO A LA REALIDAD LOCAL.}

\section{Características de las Políticas Públicas.}

Antecediendo a la presentación y posterior análisis de la especificidad en el tratamiento público social en la órbita uruguaya es menester presentar algunos conceptos desde un nivel general; en el entendido de que cuando 
hablamos de intervención pública salimos de la esfera privada; y haciendo las mediaciones correspondientes podríamos definirla diciendo que la misma “... pretende resolver un problema social reconocido políticamente como público. La definición propuesta supone pues el reconocimiento de un problema, es decir, la existencia de una situación de insatisfacción social, cuya solución requiere la acción del sector público" (Subirats; et al. 2008, p. 37). En relación a lo anterior, por lo tanto, entendemos que las políticas públicas que tienen por objeto de intervención los problemas sociales y en las cuales el Estado interviene son denominadas políticas sociales,

Las políticas públicas que suelen ser presentadas como sociales, en la mayor parte del mundo capitalista contemporáneo, incluyen a todas aquellas intervenciones públicas que regulan la forma en que la población se reproduce y socializa, ... y que además protegen a la población de situaciones que ponen en riesgo esos procesos o neutralizan los efectos $<<$ no deseados $>>$ de los mismos." (Andrenacci y Repetto, 2006, p. 2)

Estas políticas, según el alcance que quieran tener, suelen subdividirse en universales y focalizadas. En cuanto al carácter universal, se hace referencia a una homogeneización de los servicios brindados, abiertos a todos los ciudadanos, donde se propagan valores, y prácticas que tienen como objetivo la igualdad social (Franco, 1996, p.17). Los ejemplos más claros de la política social universal son la salud, la educación, entre otras. Por su parte, el concepto de focalización nos remite a la realización de una discriminación positiva a cierta parte de la población con el objetivo de paliar ciertas problemáticas específicas que se reflejan en el seno de una población determinada (Franco, 1996, p.18). Los Estados, mediante el desarrollo de las políticas públicas y sociales, universales y focalizadas, procuran minimizar la brecha social en el acceso a la oferta pública, logrando como consecuencia que la población acceda a los aparatos de protección social; por tanto, se puede sostener que la política social debería buscar la ampliación de la ciudadanía, la misma es la "... noción que define la pertenencia de los individuos a las organizaciones político-territoriales. En el mundo contemporáneo, ... la ciudadanía implica la pertenencia de un individuo a un Estado-nación" (Andrenacci y Repetto, 2006, p. 4).

A partir de lo anterior se desprende, desde un punto de vista político, que la ciudadanía representa una categoría que resulta del proceso de conquista, social, político y económico en el desarrollo de la sociedad capitalista. $^{2}$

2 Los conceptos presentados fueron escogidos a criterio de quien escribe según los objetivos del presente documento, pero los mismos han sido estudiados y definidos por innumerables autores. 


\section{BREVE PERIODIZACIÓN DE LAS POLÍTICAS SOCIALES EN URUGUAY}

El Estado uruguayo ha presentado diferentes momentos en lo que refiere a la planificación y gestión de políticas sociales tanto universales como focalizadas. Haciendo un recorte temporal necesario para los objetivos del documento, notamos que a partir de la segunda mitad del siglo XX se reflejan avances y retrocesos en la intervención pública.

Los años 50' caracterizados por el modelo neobatllista ${ }^{3}$ con un enfoque social sostenido en una fuerte intervención del Estado, de gran impulso al desarrollo industrial y una época de bonanza económica, representaron grandes avances en la política social. Hacia la década del 60,'en cambio, comienza a visualizarse un declive en el escenario social con un modelo de industrialización agotado que marcó el fin del Estado de bienestar uruguayo. Esta coyuntura se acompaña durante la década del $70^{\circ}$ y $80^{\prime}$ de la dictadura cívico militar y el retiro completo del Estado 4 . Los años 90, por su parte, se inician con el modo de acumulación capitalista como triunfador bajo las directrices planteadas en el Consenso de Washington. Tanto a nivel mundial como local se instaló el modelo neoliberal dando paso a la regulación de los Estados a través de los mercados mundiales retirando, o promoviendo en menor medida la intervención pública de los países, un ambiente propicio para la generación y aumento de la pobreza.

La privatización de la oferta pública y la regulación mediante la economía mundial ponen fin al welfarestate ${ }^{5}$. Bajo esta coyuntura económica, social y política, reaparece el concepto de cuestión social para el cual Iamamoto (1997) expresa lo siguiente,

... no es otra cosa que expresiones del proceso de formación y desarrollo de la clase obrera y de su ingreso en el escenario político de la sociedad, exigiendo su reconocimiento como clase por parte del empresariado y del Estado. Es la manifestación, en el cotidiano de la vida social, de la contradicción entre el proletariado y la burguesía, la cual pasa a exigir otros tipos de intervención, más allá de la caridad y represión. (p.92)

Frente a este escenario de neoliberalismo mundial, “... las respuestas de nuevas políticas sociales partieron del supuesto de tratar estos fenómenos

3 El término hace referencia al político perteneciente al partido colorado, Luis Batlle Berres (1897-1964) caracterizado por desarrollar una política estatista y proteccionista en sus años de presidencia, de 1947 a 1951.

4 Si bien en este documento se hace referencia a la realidad uruguaya, este recorrido histórico también es aplicable a los demás países del cono sur los cuales sufrieron terrorismo de estado en igual período.

5 Esta denominación hace referencia al término "Estado de Bienestar", acuñado luego de la segunda guerra mundial. 
como transitorios de efectos económicos disfuncionales y focalizados en algunos sectores de la sociedad, procurando optimizar la eficacia de la intervención pública <<las denominadas redes de seguridad >>" ( Midaglia, Serna, Rivero citado por Cerutti, et al. 2014, p.7).

Este nuevo enfoque en el tratamiento de las políticas sociales procuraba intervenciones breves, concretas, desde la postura teórico política de que la brecha social respondía a disfuncionalidades generadas por el mercado, por tanto, se dejó a un lado la historicidad y las trayectorias de los procesos sociales. Como consecuencia, no se minimizaban las inequidades, no se transformaban las condiciones de vida de los sectores más desfavorecidos y no se reducía la desigualdad social. Las políticas sociales se transformaron en subsidiarias de las políticas económicas; continuando en la misma línea de las autoras notamos que,

... el crecimiento económico fue menor de lo esperado mientras que la mejora de las condiciones de vida del conjunto de la población fue inexistente, registrándose, por el contrario, un aumento del nivel de pobreza y una mayor desigualdad en la distribución del ingreso. Hacia los 90's se entendía que la estabilidad macroeconómica y las políticas a favor de la reapertura de los mercados eran insuficientes para resolver problemas agudos como el de la pobreza. (Cerutti, et al. 2014, p.8)

Cuando comienza el nuevo milenio, Uruguay no presenta grandes cambios respecto a los 90’ en el plano económico,

... la recesión de 1999 a 2003 y su clímax en 2002, estas últimas productoras de incrementos significativos en los niveles de pobreza y la desigualdad... uno de los mayores problemas que la economía uruguaya ha experimentado... ha sido la elevada volatilidad de su comportamiento (De Armas, 2009, p.34-35).

Estos comportamientos en la economía acarrean también efectos en la esfera social, "... aumentos abruptos en las tasas de pobreza, irrupción de procesos de exclusión social, incremento de la propensión migratoria y afirmación de los comportamientos reactivos al riesgo..." (De Armas, 2009, p. 35).

Este escenario se presenta como antesala en los primeros años del siglo XXI cuando en el país luego de doscientos años de gobierno a cargo de los partidos tradicionales, en el 2005 asume por primera vez en Uruguay una coalición de izquierda, el Frente Amplio, que bajo su concepción progresista 
plantea en su agenda política diversas transformaciones y priorizaciones; modificando casi por completo el sistema de seguridad social generando políticas públicas de combate a la pobreza. La nueva matriz de protección desarrolla una serie de políticas tanto universales como focalizadas dirigidas a diferentes sectores de la población con el objetivo de atender la emergencia social por la que transitaba el país en ese momento, ejemplo de ello es el fenómeno de infantilización de la pobreza, en el año 2003 el Instituto Nacional de Estadística "había estimado que el 56,5\% de los menores de 6 años eran pobres y el 13,3\% indigentes." (Grau, 2005, p. 88) Estas políticas se desarrollaron prácticamente en su totalidad mediante la adjudicación en la gestión a la sociedad civil y, en menor medida, mediante la gestión directa del estado (actualmente se continúa bajo este sistema mixto).

Una de las primeras acciones de carácter universal que el gobierno recién asumido implementa es la creación en el año 2005 del Ministerio de Desarrollo Social, cartera a la que se le concede la gestión y articulación de los programas y dispositivos creados para enfrentar la emergencia social que atravesaba el país. Las dos acciones principales la constituyen el Plan de Atención a la Emergencia Social (PANES) el cual comprende la primera etapa y se plasma a través de la Ley 17.869. Se trata de una política claramente focalizada que se caracteriza por dar prioridad a la atención de las personas en situación de extrema vulnerabilidad social, a través de respuestas inmediatas que, de acuerdo al gobierno, no podían dilatarse en el tiempo. Comienza a implementarse en marzo del 2005 y finaliza en diciembre del 2007, donde se engarza con el Plan de Equidad. (Cerutti, et al. 2014, p.15-16)

El sucesor, Plan de Equidad (PE),

Consiste en una estrategia de mediano y largo plazo orientada a actualizar la matriz de protección existente, a partir del diagnóstico de los nuevos problemas sociales y de la identificación de los soportes y malla de protección social preexistentes. Define como objetivos estratégicos:

1. Mejorar los niveles de equidad entre generaciones, géneros y etnias; 2. Consolidar y ampliar una red de asistencia social con la finalidad de impedir aumentar la cronificación de situaciones de pobreza e indigencia;

3. Articular dicha red al conjunto de prestaciones de las que el país ya dispone, conformando así un único y renovado sistema de protección social (Cerutti, et al. 2014, p.20-21). 
Cabe destacar que el Ministerio recién creado manifestaba un fuerte componente territorial, apostaba a ser un "gobierno de cercanía" a partir del trabajo bajo este tipo de intervenciones, por tanto, se descentralizó a nivel país mediante 19 direcciones departamentales (una por departamento) y desde allí, conformado por una diversidad de programas sociales, se comenzó a ejecutar la política social. Si bien en la especificidad la política social presentó ciertos cambios con el transcurrir de los años, desde un nivel general, no hubo transformaciones estructurales de impacto.

\section{POLÍTICAS PÚBLICAS DE PROTECCIÓN A LA INFANCIA Y ADOLESCENCIA EN URUGUAY.}

En este apartado se intentará ser aún más específica por lo que se presentará de forma clara y resumida el recorrido que han tenido las políticas de infancia y adolescencia a partir de los marcos normativos que ha tenido cada época. ${ }^{6}$ Se irá hacia adelante y atrás en el tiempo invitando a las y los lectores construir y deconstruir la categoría infancia y adolescencia.

Previo al análisis es menester presentar el enfoque de las categorías mencionadas,

... parece haber un acuerdo mínimo en que la categoría infancia fue construyéndose a través de la historia, ya sea mediante una revolución o a través de un cambio paulatino. ... La importancia de la figura del niño como actor social en la actualidad es indiscutible y el intento por comprender su historia forma parte de la tendencia a reconocer su singularidad (González, 2011, p.20).

Entendiendo a la infancia como parte del tejido social, Nieves (2004) invita a su análisis desde diferentes dimensiones;

Todos los cambios sobre la percepción de la infancia no se desarrollan de forma aislada, por el contrario, hay que comprenderlos dentro de los procesos sociales más amplios, de índole económica, política, social y cultural. Los modelos y el lugar social de los niños ha cambiado históricamente, en estrecha relación con los cambios de actitud de los adultos frente a los niños (Nieves, 2004, p.152).

6 Para los objetivos del presente documento no es de relevancia la época previa a la década de 1930', de todos modos, se recomienda el presente autor para los/as lectores interesados/as en la historia de la representación social de la infancia en Uruguay: José Pedro Barrán (1990): Historia de la sensibilidad en el Uruguay: La cultura "bárbara" (18001860). El disciplinamiento (1860-1920). Montevideo: Ediciones de la Banda Oriental, 441 p. 
Aterrizando en el escenario histórico uruguayo, se partirá del año 1933 en el cual se decreta la conformación del Ministerio de Protección a la Infancia el cual tendrá como objetivo principal reorganizar, así como ordenar todos los dispositivos y servicios públicos vinculados a la infancia. Este es un elemento que representa la antesala de lo que será el Código del Niño, sancionado en 1934, teniendo en cuenta que, en sintonía con la conformación del Ministerio, se conforma en paralelo una Comisión Redactora de la Legislación del Menor. (Leopold, 2002, p.45) Es el año mencionado, 1934, que marca otro punto de inflexión en Uruguay. A nivel normativo e institucional se sanciona el Código del Niño y se crea el Consejo del Niño. Se intentará tomar- a partir de este momento- a la categoría social infancia desde una perspectiva integral; ensamblándose el accionar de diferentes instituciones, de diferentes áreas.

El 6 de abril de 1934, en acuerdo con el Ministro de Protección a la Infancia, Gabriel Terra7 puso el "cúmplase" al Decreto Ley que daba su aprobación al Código del Niño, a la vez que fundaba el Consejo del Niño, en aplicación del Art. 1o de dicho Código, como organismo especializado, exclusivo y excluyente (Turnes y Rovira, 2014, p. 49).

En los años posteriores a 1934 Uruguay transitó por un Estado de bienestar; intervencionismo estatal, políticas sociales que se ampliaban en todo el territorio nacional logrando el universalismo, especialmente, si nos referimos a niños y niñas se destacó la educación y la salud. "La estructura de Bienestar Uruguaya se caracterizó por una mezcla de servicios de tendencia universalista para aquellos integrados al mercado formal; y, por otra parte, focalización para los sectores más pobres y sin capacidad de acceso al mercado." (Castillo y Milanesi, 2014, p. 62)

Las décadas subsiguientes a las mencionadas (presentadas en el apartado anterior) si bien presentaron transformaciones desde un punto de vista social en términos puramente institucionales de acciones dirigidas a la infancia no presentaron cambios significativos hasta la década de los 70' momento en el cual se quiebra el tejido social cuando irrumpen en toda la región dictaduras cívico- militares como ya se mencionó anteriormente.

Pese a suspender la ciudadanía política y con ello las demandas distributivas, el gobierno de facto no introdujo cambios sustantivos en los lineamientos económicos y sociales... No obstante, se utilizaron instrumentos de depresión de las políticas de seguridad social... que

7 Gabriel Terra, político uruguayo que perteneció al partido colorado de ese país, en ese entonces Presidente de Uruguay. 
si bien no revirtieron la matriz esencial de bienestar... resintieron la calidad y el nivel de los servicios y prestaciones sociales (Midaglia, 2000, p. 27).

Con la democracia restituida, ya a fines de la década de los 80 , este contexto se caracterizó por la aprobación de la Ley 15.977 la cual sustituye al Consejo del niño por el INAME (Instituto Nacional de Menores). Esta ley lo que hace es otorgarle a dicha institución mayor grado de autonomía, respecto a la administración central;

...este período puede caracterizarse por la consolidación de la institucionalidad dirigida a la infancia. En términos de la orientación general del modelo, el mismo combinó una tendencia universalista e integradora en el caso de la educación y otra orientada a la focalización en grupos vulnerables para las políticas de protección (Castillo y Milanesi, 2014, p. 63).

Con estenuevo escenario, el INAME comienza a revisar sus lineamientos y los programas específicos que desarrolla en la protección a la infancia. La transformación en un servicio descentralizado permitió pensar y ampliar el abanico de programas, políticas y recursos destinados a la infancia.

Al igual que otros servicios públicos, asumió nuevas problemáticas sociales y en consecuencia programas específicos, e inició un conjunto de tercerizaciones tendientes a redefinir su relación con los sectores privados, en particular con las instituciones civiles que desarrollaban acciones en este campo (Midaglia, 2000, p. 30) ${ }^{8}$.

Estas modificaciones que se realizaron también se reflejaron en el punto de vista teórico de la categoría infancia. En el año 2005 se aprueba el Código de la Niñez y la Adolescencia cambiándose la concepción jurídica y el Instituto Nacional del Menor (INAME) pasa a denominarse Instituto del Niño y Adolescente del Uruguay (INAU). Esta institución, de acuerdo con la Ley 17.866, está vinculada al Poder Ejecutivo a través del Ministerio de Desarrollo Social. ${ }^{9}$

El Código de la Niñez y la Adolescencia que rige actualmente en Uruguay está conformado por 224 artículos, enmarcado bajo la normativa

8 Es pertinente destacar entre los nuevos programas la creación de los Centros de Atención a la Infancia y a la Familia (Plan CAIF). Esta estrategia, vigente en la actualidad forma parte de la combinación público privado que se comenzó a desarrollar; el Estado y la Sociedad civil.

9 Para ampliar la información presentada se recomienda ver la página web del Instituto del Niño y Adolescente del Uruguay. Disponible en: https://www.inau.gub.uy/institucional/historia 
establecida en los principios de la Convención y en el paradigma de la protección integral. Plantea una serie de diferencias con el código de 1934 el que se sustentaba en la situación irregular de la infancia y adolescencia. No es el objetivo de este trabajo analizar el actual código en términos jurídicos, pero sí hacer una puntualización que se considera la base fundamental de lo que se expone.

Si bien el marco regulador internacional en el que se sostiene el Código es la Convención, es pertinente señalar que cada país tiene su especificidad económica, política y geográfica, lo que impacta directamente en el gasto público social destinado a la infancia y adolescencia. Dicho esto, en algunas ocasiones en Uruguay se visibiliza una gran distancia cuando se intenta aplicar al escenario local. Ejemplo de ello es que si existe una falta de robustez en el Estado, el cual se considera que es quien debería ser el promotor en el acceso a la matriz social - no asíel sector privado para la generación de políticas sociales de impacto en la infancia y adolescencia-, nos enfrentamos en ocasiones a una brecha en términos de aplicabilidad de los artículos del Código.

El actual Código uruguayo postula en sus artículos, a los niños, niñas y adolescentes como sujeto de derechos, deberes y garantías. Esta es quizás una de las diferencias más grandes con el anterior código. No son objeto de protección desde la pasividad, sino que bajo el Estado de Derecho en el cual se encuentran, también les corresponden deberes y obligaciones. A grandes rasgos, este código tiene el objetivo de la protección a la infancia transversalizado con la actuación de diversas instituciones y organismos. En este punto, nuevamente cabría preguntarse si las políticas y específicamente los programas de protección a la infancia y adolescencia actualmente se ejecutan a partir de los principios que establece el código o si continúan los vestigios de la situación irregular.

Esta problematización se podría analizar desde diversas aristas: a) desde la profesional, indagando bajo qué paradigma se interviene con los niños, niñas y adolescentes; b) desde lo presupuestal, si el sistema de protección cuenta con los recursos que debería; y c) desde lo interinstitucional, indagando acerca de la implicancia que tienen los organismos que trabajan con la infancia. Algunas de estas dudas serán clarificadas en las conclusiones del documento, y otras quedarán como interrogantes para futuras investigaciones. Lo que sí es menester y no se puede dejar de señalar es que la infancia en términos de gasto público desde los primeros años del nuevo milenio tomó cierto protagonismo. 
El incremento en términos absolutos y relativos del GPSI ${ }^{10}$ y del GPSI por niño y adolescente ha sido resultado, básicamente, de cuatro factores: a) la implementación del Plan Nacional de Atención a la Emergencia Social (PANES) entre 2005 y 2007; b) el crecimiento constante del gasto público en servicios educativos dirigidos a niños y adolescentes ... c) la reforma de la salud a partir de 2008 (la incorporación de los niños y adolescentes al Sistema Nacional Integrado de Salud); y d) la implementación del nuevo Régimen de Asignaciones Familiares - Ley 18.227- también desde 2008 (Arroyo, et al. 2009, p. 16,17).

Continuando el recorrido de la agenda política uruguaya y la priorización a las categorías infancia y adolescencia, en el año 2015, mediante la Ley $\mathrm{N}^{\circ} 19.353$ con fecha de aprobación el 18 de noviembre y promulgación el 27 de noviembre, establece que en el país todas las niñas y niños, personas con discapacidad y personas mayores en situación de dependencia tienen derecho a ser cuidadas; de esta manera, se crea el Sistema de Cuidados. Esta estrategia tiene el objetivo de generar un modelo corresponsable en materia de cuidados, vinculando a las familias, al Estado, a la comunidad y al mercado. El otro eje vertebrador es la responsabilidad compartida del cuidado entre hombres y mujeres, tarea históricamente asignada a las mujeres, por lo que el sistema de cuidados adopta una perspectiva de género.

Finalizando el racconto histórico, se presenta otro eje priorizado en la intervención pública social destinada a infancia y adolescencia. En el año 2019, a partir de la reforma al Código de la niñez entra en vigencia la Ley $\mathrm{N}^{\circ} 19747$ la cual consagra la creación del SIPIAV (Sistema Integral de Protección a la Infancia y a la Adolescencia contra la Violencia) la cual funciona en la órbita del Instituto del Niño y Adolescente de Uruguay quien también lo preside. ${ }^{11}$ Si bien el SIPIAV fue creado en el año 2007 por diversas instituciones públicas que abordaban la temática y procuraban establecer acuerdos mínimos para el abordaje de las situaciones de violencia en la infancia y adolescencia, el Sistema no contaba con un marco jurídico.

La Ley N 19747 establece los cometidos que va a tener el SIPIAV, estos son:

1 a. Prevenir, atender y reparar las situaciones de violencia hacia NNA mediante un abordaje integral e interinstitucional.

b. Promover modelos de intervención desde las distintas instituciones para asegurar la integralidad del proceso. (Tomado de la Ley $\mathrm{N}^{\circ}$ 19747).

10 Gasto Público Social en Infancia.

11 Por más información del SIPIAV, dirigirse al siguiente link: https://www.inau.gub.uy/sipiav 
Hasta aquí se abordó la temática desde lo universal a lo particular, presentando mediante un breve recorrido las principales acciones que tomó el estado uruguayo en materia de planificación y gestión de políticas públicas dirigidas a infancia y adolescencia. En el próximo y último apartado se analizará la situación actual de tres dispositivos de acompañamiento familiar.

\section{DESAFÍOS Y TENSIONES EN PROGRAMAS CON METODOLOGÍA DE PROXIMIDAD EN INFANCIA Y ADOLESCENCIA}

\section{Cercanías - Uruguay Crece Contigo - Jóvenes En Red.}

Los tres programas implementados desde el año 2012, fueron concebidos como una estrategia de abordaje integral colocados en la agenda pública como "programas prioritarios" con anclaje territorial. "Su génesis fue simultánea y respondió a la necesidad de construir respuesta pública a los problemas sociales coyunturales, a su vez, presentan similitudes metodológicas importantes en lo que refiere a sus modelos de atención, de gestión y mecanismos de selección de la población objetivo" (Lasa, 2005:12).

Estos dispositivos se configuraron a partir de experiencias internacionales, los mismos comenzaron a desplegarse alrededor de todo el Uruguay con el objetivo de que las familias en situación de extrema vulnerabilidad pasen a ocupar el centro en lo que refiere a la oferta pública de prestaciones bajo la perspectiva de derechos. Cercanías, se enfoca en el trabajo de acompañamiento con todo el núcleo familiar; Uruguay Crece Contigo, se focaliza en mujeres embarazadas y la primera infancia y Jóvenes en Red, se enfoca en el trabajo con la población adolescente.

En cuanto al primer programa mencionado, y a partir del material recogido teóricamente al programa Cercanías, lo definen de la siguiente forma:

... es una iniciativa interinstitucional que se plantea trabajar con grupos familiares que presentan sus derechos vulnerados estando en situación de extrema pobreza, durante un período máximo de 18 meses utilizando metodología de proximidad para trabajar en función de metas concretas de inclusión social- (Rieiro, et al.2015, p. 7).

Este programa estableció un despliegue territorial a nivel país con el Ministerio de Desarrollo Social como centralidad y con acuerdos interinstitucionales con otras carteras, ejemplo de ello: Ministerio de 
Vivienda, Ministerio de Salud, Ministerio de Educación, entre otros. Los equipos tenían el objetivo de funcionar acercando a los núcleos familiares en situación de extrema pobreza a la oferta pública social.

Otro de los grandes programas que se impulsaron, con focalización en la primera infancia, fue el programa "Uruguay Crece Contigo"12, el mismo recoge la trayectoria internacional de "Chile Crece Contigo" y a nivel local de "Canelones Crece Contigo". Dicho programa tiene como objetivo la protección a la primera infancia mediante un sistema de protección integral tanto con acciones universales como focalizadas. La población objetivo del programa son mujeres embarazadas, así como niños y niñas menores de cuatro años. Los acompañamientos son en modalidad de cercanía con perspectiva de derechos, género y generación.

Por último, el Programa Jóvenes en Red, también dependiente del Ministerio de Desarrollo Social, surgió como otra de las estrategias en política focalizada a adolescentes y jóvenes en situación de vulnerabilidad, desvinculados tanto del sistema educativo como del mercado laboral formal. La cobertura comprendió las edades de 14 a 24 años y se propuso "... un trabajo en la comunidad mediante la integración articulada de cuatro componentes: social, educativo, laboral y de fortalecimiento comunitario." (Ministerio de Desarrollo Social [MIDES], s.f.)

El programa también se despliega con modalidad de cercanía acompañando a los y las jóvenes en sus procesos, sus preferencias y sus necesidades personales buscando promover la autonomía y su propio proyecto personal.

Hasta aquí, se presentaron brevemente los tres programas sociales dirigidos a infancia, adolescencia y familia. En adelante, se intentará analizar qué sucede con el Estado y su gestión de la política pública frente a un cambio de gobierno.

En el año 2020 asume en el país un nuevo gobierno: la derecha, y plantean en su agenda política la generación de transformaciones en la órbita pública social, específicamente en los programas dependientes del Ministerio de Desarrollo Social. Se proyectan rediseñar los dispositivos que se venían desplegando hasta ese momento. En palabras de quien asume la Dirección General de la cartera ministerial se manifiesta lo siguiente:

12 La página web del Programa se encuentra en el siguiente enlace: https://www.gub.uy/ministerio-desarrollo-social/uruguay-crece-contigo-ucc 
...el programa Cercanías se va a fusionar con el programa Uruguay Crece Contigo -de protección integral a la primera infancia- porque son dos programas de territorio "que abarcan el tema familia" y que, por lo tanto, se entiende que deben ser abordados de formas similares ... el programa va a seguir funcionando igual en sus objetivos, pero "con mucho más criterio", porque Cercanías de la forma en que estaba funcionando no estaba dando los resultados que las nuevas autoridades entienden que debía dar. (La Diaria, 08 de mayo de 2020)

Por su parte, desde otros sectores, y quienes trabajan en contacto directo con la población destinataria de estas políticas, se manifiestan otro tipo de posturas. Se habla de desmantelamiento de los programas sociales, “... repliegue de los programas del $\mathrm{Mides}^{13}$ en el territorio y un progresivo desmantelamiento de los servicios sociales, y pone como ejemplo Jóvenes en Red, Socat ${ }^{14}$, Tránsito Educativo y los Centros Promotores de Derechos, entre otros programas". (La Diaria, 01 de mayo de 2021)

En otra fuente de texto escrito, quien fuera subsecretaria en la anterior administración sostiene: "Esta primera etapa de la gestión se encargó de desarmar programas, y en vez de sustituirlos por otros, los enlenteció, discontinuó. Cuando no hay un diálogo y una construcción colectiva, es muy difícil generar políticas públicas." Vila (03/05/2021) Ana Olivera, sobre el Mides: "Fue el año del desmantelamiento de las políticas sociales" y de "desarmar programas". (El Observador 03 de mayo de 2021)

Pasado más de un año de la asunción de las nuevas autoridades, con las transformaciones y/o rediseños como se menciona, la realidad es que la política con metodología de proximidad está en un momento clave ${ }^{15}$. Es de orden señalar que no es a los objetivos de este documento presentar la situación en términos de los convenios que mantiene el estado con la sociedad civil mediante el mecanismo de tercerización para la ejecución de los programas sociales, sino analizar desde un nivel más general la relación de la política pública destinada a infancia y adolescencia, y el compromiso a partir de los acuerdos firmados a nivel internacional.

Uruguay es uno de los 195 estados que ratificó la Convención sobre los Derechos del Niño (en adelante la CDN), la misma, establece según lo analizado lo siguiente:

13 Ministerio de Desarrollo Social.

14 Programa perteneciente al Ministerio de Desarrollo Social el cual realizaba un servicio de orientación, consulta y articulación territorial, de allí su sigla SOCAT. El mismo finalizó en el mes de noviembre en todo el territorio nacional.

15 Sumado a la situación mundial sanitaria que imposibilitó prácticamente la circulación, replegó a la población al encierro y generó grandes pérdidas en el empleo. 
Todos los derechos de los niños están recogidos en un tratado internacional que obliga a los gobiernos a cumplirlos: la Convención sobre los Derechos del Niño (CDN). Es el tratado más ratificado de la historia y los 195 Estados que la han ratificado tienen que rendir cuentas sobre su cumplimiento al Comité de los Derechos del Niño. Los 54 artículos que componen la Convención recogen los derechos económicos, sociales, culturales, civiles y políticos de todos los niños. ... El Comité de los Derechos del Niño está formado por 18 expertos en derechos de infancia procedentes de países y ordenamientos jurídicos diferentes. (UNICEF, 2006).

A partir de lo anterior, se decanta que los estados que han ratificado la $\mathrm{CDN}$ se deben regir por los principios orientadores de la misma, esto es a nivel: jurídico, económico y social; y que el Comité es el organismo encargado de evaluar en cada país el cumplimiento o no de los artículos establecidos. Desde un nivel más universal a lo particular, nos enfrentamos a que cada país establece su aplicabilidad mediante su código propio ${ }^{16}$ el cual aterriza y direcciona en la especificidad de cada contexto. A más de treinta años de la Convención, se reflejan ciertos avances en diferentes dimensiones del campo social en lo que refiere al acceso a la salud, a la educación; así como avances en materia legislativa.

Se han realizado importantes cambios en las normas jurídicas y en las políticas sociales públicas destinadas a hacer realidad los derechos contenidos en el Documento de la Convención. Logros y políticas que, impulsados por los Estados o por las organizaciones humanitarias, han logrado importantes mejoras en las condiciones de vida de millones de niños ... (Guilló, 2007, p.88).

Uruguay, caracterizado por ser un país con un gran componente estatista, en los primeros años del nuevo milenio estuvo a la vanguardia en materia de creación de políticas de protección a la infancia y adolescencia generando una base de anclaje estatal que aseguró condiciones mínimas en el acceso a la matriz social. También es necesario aclarar que hay ciertos núcleos duros que mantienen una situación de pobreza estructural y que actualmente continúan en el mismo escenario, por tanto, no se trata de romantizar las situaciones de vulnerabilidad cuando, si bien se han desplegado una gran cantidad de programas de combate a la pobreza, los mismos no han dado resultados significativos; más bien, han sido una especie de "parche" temporal; por tanto, se invita a problematizar estas situaciones, analizar qué sucede con la política pública en el escenario social cuando los gobiernos de

16 En el caso de Uruguay como ya se mencionó anteriormente existe el Código de la Niñez y Adolescencia. 
turno deciden quitarla o reprogramarla, cuáles terminan siendo las garantías de que el estado va a proveer de asistencia a la ciudadanía,

... el carácter de la asistencia asume su significación más completa y compleja cuando es el Estado que toma para sí la atención de las expresiones problemáticas derivadas de la cuestión social. En este marco, la asistencia se vuelve un asunto de Estado, un asunto que demanda la participación de saberes especializados con sus agentes profesionales en un proceso de gestión burocrática de los recursos públicos y las necesidades/demandas sociales (Pérez y Vecinday, 2016, p.93).

A partir de lo anterior, también se desprende otro nivel de análisis: si los programas que trabajan con metodología de proximidad realmente pueden generar movimientos y ser agentes de cambio en las situaciones con las que se interviene o si se terminan generando espacios de escucha más que transformaciones reales de las condiciones materiales.

Parece absurdo pensar que, ante las situaciones más críticas de pobreza extrema y fragilidad familiar, un "acompañamiento inmaterial" sea suficiente para revertir lo que décadas de exclusión y desigualdad han venido construyendo ... En programas de este estilo parece depositarse excesivas e irreales expectativas de lo que puede la intervención directa cara a cara, la tarea socioeducativa desprovista de la gestión directa del recurso material de la asistencia (Pérez, Vecinday, 2016, p.99).

Por lo tanto, y a partir de lo anterior nos encontramos con un nudo problemático que refiere a la falta de materialidad en las intervenciones, las cuales son imprescindibles para pensar en un proceso mínimamente transformador de realidades que son producto de situaciones de pobreza estructural. "La carencia de estos recursos materiales, conlleva a pensar en un proceso de desmaterialización de las políticas y al peligro de "psicologizar" los problemas sociales estructurales, responsabilizando al sujeto por las dificultades que expone su exigencia" (Leopold y González, 2015:222).

La presentación de los tres programas escogidos como ejemplo y el aporte teórico intentan ser una primera aproximación a la discusión acerca del alcance y los límites que tienen los dispositivos de cercanía; no obstante, se invita a reflexionar acerca de la importancia de que los estados se hagan cargo de la asistencia generando programas que se puedan sostener en el tiempo al margen de los gobiernos de turno. En acuerdo con Pérez y Vecinday (2016) "Creemos que el debate sobre la política social debe girar en torno a la orientación general de la intervención social del Estado..." (Pérez, Vecinday. 2016, p.94) 
Si bien es ilusorio pensar que con la sola intervención de una única política focalizada desprovista de materialidad se pueda resolver la condición de vulnerabilidad en sí misma, sí se puede llegar a transformar mínimamente la gravedad de esa situación; y si se agrega que estos acompañamientos permitan la flexibilidad de ser más duraderos en el tiempo pueden habilitarel accesoreal a los diferentes recursospúblicos.

\section{CONCLUSIONES}

1. A lo largo del documento se fueron presentando los cambios en el sistema de protección social uruguayo, y específicamente en la gestión de las políticas sociales vinculadas a infancia y adolescencia. Se analizaron las transformaciones suscitadas en los primeros años del nuevo milenio, el recorrido de las mismas, y finalmente, se aterrizó en el escenario político social de ascenso de las nuevas autoridades en marzo de 2020 y las incipientes transformaciones que se están gestando en materia de intervención pública social. Es necesario mencionar que, al abordar una temática tan cercana en el tiempo, se refleja la falta de respaldo teórico. Si bien el tratamiento de la intervención pública está más que estudiado, no así el abordaje de la temática específica que se presenta: la finalización del mandato de la izquierda y el ascenso de la derecha en Uruguay y como eso se materializa en la arena social. Por lo tanto, este documento intenta ser un insumo para continuar en el estudio de la temática.

2. De lo presentado surge, por un lado, la necesaria evaluación crítica acerca de los alcances y las limitaciones que tienen los programas con metodología de proximidad. Por el otro, la evaluación acerca de si como agentes de cambio que realizamos nuestra praxis en el área social somos lo suficientemente críticas/os, en si tenemos la posibilidad de mínimamente generar procesos de transformación real en las situaciones que abordamos las cuales son en su mayoría situaciones familiar esa las que se les han vulnerado históricamente sus derechos. Podríamos citar a De Martino (2014) quien plantea qué papel juegan en materia de políticas sociales los avances legislativos, ¿realmente se traducen al escenario social?

La dosis de violencia, material y simbólica, que se reflejan en los niveles de pobreza de la población beneficiaria de los servicios analizados, en las problemáticas que atraviesan, y en el tipo de respuesta institucional que reciben con relación a la magnitud de sus necesidades, más allá de buenas intenciones, parecerían ser 
activamente aceptadas, pasivamente contempladas por la sociedad en su conjunto (De Martino, 2014 p. 71).

De aquí se desprende la importancia de la existencia de un Estado presente que pueda hacerse cargo de la asistencia a las personas en situación de extrema vulnerabilidad.

3. En particular, con las categorías infancia y adolescencia, para lograr y garantizar la protección real de niñas, niños y adolescentes, debe contar la intervención pública con un gasto social significativo, desplegando recursos tanto materiales como humanos que duren en el tiempo y que garanticen abordajes de calidad. De lo contrario, los estados pueden ratificar numerosas convenciones internacionales, pero sin generar praxis reales y significativas.

\section{REFERENCIAS}

Alegre, P. y Filgueira, F. (2009). Una reforma híbrida. La política social y laboral en Uruguay de 1985 a 2008. Revista Internacional del Trabajo, núm. 128 (3), 349-367.

Andrenacci, L y Repetto, F (2006). Universalismo, ciudadanía y Estado en la política social latinoamericana. [en línea]. CIESU, Centro de Informaciones y estudios del Uruguay. http: / / ciesu.edu.uy / sitio_viejo / universalismo/Andrenacci.pdf

Arroyo, A, et al. (2009). Observatorio de los derechos de la infancia y la adolescencia en Uruguay 2009. Montevideo: Fondo de las Naciones Unidas para la Infancia, UNICEF.

Castillo, M y Milanesi, A (2014). La institucionalidad pública de infancia en Uruguay: evolución histórica y nudos críticos para el debate. Montevideo. Publicaciones del instituto de estudios legales y sociales del Uruguay (IELSUR), Ciudadanía, niñez y adolescencia, p. 59-78.

Cerutti, A, et al. (2014). Políticas sociales para la infancia en el Uruguay con énfasis en las edades tempranas. Propuestas de monitoreo del bienestar infantil. Documento de trabajo $\mathrm{N}^{\circ} 2$, Centro interdisciplinario de infancia y pobreza, espacio interdisciplinario. Universidad de la República, ISBN: 978-9974-0-1092-5. 
De Armas, G (2009). Primero la infancia. Desarrollo, Estado y políticas sociales en Uruguay. Montevideo: CLAEH Centro Latinoamericano de Economía Humana.

De Martino, M (2014). Políticas de protección a niños y adolescentes en América Latina.Revista Regional de Trabajo Social. Vol. 28. N60. Uruguay. ISSN: 0797-0226. Pp. 58-77

Franco, R (1996). Los paradigmas de la política social en América Latina. Chile: Revista de la CEPAL (Comisión Económica para América Latina y el Caribe), No 58, P. 9-22.

González, M (2011). Derechos Humanos de los Niños: Una propuesta de fundamentación. Universidad Nacional Autónoma de México. Instituto de investigaciones jurídicas, serie Doctrina Jurídica. Núm. 425, 533 p.

Grau, C (2005). Inversión en la infancia en Uruguay. Análisis del gasto público social: tendencias y desafíos. El gasto público social en infancia en Uruguay. Aspectos metodológicos y cuantificación para el período 1999-2002. Fondo de las Naciones Unidas para la Infancia, UNICEF, 199p.

Guilló, J (2007). La Convención sobre los Derechos del Niño. Derechos y Necesidades de la Infancia. En: HERNÁNDEZ, Manuel, et al. Los Derechos de los Niños, responsabilidad de todos. Murcia: Universidad de Murcia, servicio de publicaciones. I.S.B.N. 978-84-8371-655-7. p. 83-95.

Iamamoto, M (1997). Servicio social y división del trabajo. Cortez editora.

Kelder, J (2005). UsingSomeoneElse's Data: Problems, Pragmatics and Provisions [56 paragraphs]. Forum: Qualitative Social Research [Online Journal], 6(1), Art. 39. https://www.qualitative-research.net/ index.php/fqs/article/view/501/1078

Lasa, N (2015). El Estado y el "Otro": reflexiones a la luz de la nueva generación de políticas sociales en Uruguay. Universidad de la República. Facultad de Psicología.

Leopold, S (2002). Tratos y Destratos: Políticas públicas de atención a la infancia en el Uruguay (1934-1973). [en línea]. Publicaciones de la Administración Nacional de Educación Pública, tesis de Maestría, 2002, 117 p. www2. convivencia.edu.uy/web/wp-content/uploads/2013/12/Tratados-yDestratados.pdf 
Leopold, S; González, C. (2015). Los riesgos de la individualización en el nuevo modelo de protección social uruguayo: el caso del Programa Jóvenes en Red. Sociedade em Debate, 21(1), 211-254.

Midaglia, C (2000). Alternativas de protección a la infancia carenciada. La peculiar convivencia de lo público y privado en el Uruguay. Publicaciones del Consejo Latinoamericano de Ciencias Sociales, CLACSO, 191 p.

Nieves, C (2004). La construcción social de la infancia. Apuntes desde la sociología. Tenerife: Publicaciones de la Revista Témpora, Universidad de la Laguna, p.149-168.

Pérez, L; Vecinday, L (2016). De la Reconceptualización a nuestros días: contradicciones de las políticas asistenciales. Revista Fronteras $N^{\circ}$ 9. Departamento de Trabajo Social, Facultad de Ciencias Sociales, Universidad de la República. ISSN: 0797-8952.

Ravetllat, I (2015). Aproximación histórica a la construcción sociojurídica de la categoría infancia. Ediciones de la Universitat Politécnica de Valencia. Colección Infancia y Adolescencia, 93 p.

Rieiro, A, et al. (2015). Tensiones territoriales y abordajes multifamiliares en contextos de vulnerabilidad: reflexiones a partir del Programa Cercanías. Cuadernos de Ciencias Sociales y políticas sociales núm. 5. Universidad de la República. ISBN 2393-6266-5.

Scribano, A; De Sena, A (2009). Las segundas partes sí pueden mejores: Algunas Reflexiones sobre el uso de datos secundarios en la investigación cualitativa. Revista de Sociología, año 11 núm. 22,p. 100-118. https://www.scielo.br/j/soc/a/ S9RkZjxKB7zzVcRN3bS3jdL/?format=pdf\&lang=es [Consulta: 15 de junio 2021].

Subirats, J. et al. (2008). Análisis y gestión de políticas públicas. Ediciones Ariel S.A. 285 p.

Turnes, A; Rovira, G. (2014). 80 Años de la fundación del Consejo del Niño.Revistas Biomedicina. ISSN 1510-9747, 2014,48-52 p.http://www.um.edu.uy/ docs/consejo_delninio_rcb.pdf 


\section{FUENTES ELECTRÓNICAS}

Diario El Observador (03 de mayo de 2021). Fue el año del desmantelamiento y de desarmar políticas sociales. https:/ / www.elobservador.com.uy/nota / ana-olivera-sobre-el-mides-fue-el-ano-del-desmantelamiento-de-laspoliticas-sociales-y-de-desarmar-programas--202153141242 Visitado el 05/08/2021.

Periódico La Diaria. (08 de mayo de 2020).Mides reformula llamados para dos de sus programas y trabajadores tercerizados advierten por efectos en politicas sociales. https://ladiaria.com.uy/articulo/2020/5/mides-reformulallamados-para-dos-de-sus-programas-y-trabajadores-tercerizadosadvierten-por-efectos-en-politicas-sociales/

Periódico La Diaria. (01 de mayo de 2021).Trabajadores del Mides denuncian "repliegue" de programas y "progresivo desmantelamiento" de los servicios sociales. https://ladiaria.com.uy/politica/articulo/2021/5/ trabajadores-del-mides-denuncian-repliegue-de-programas-yprogresivo-desmantelamiento-de-los-servicios-sociales /

Ministerio de Desarrollo Social. Guía de recursos. https://guiaderecursos. mides.gub.uy/34128/jovenes-en-red .Visitado el 10/08/2021.

\section{FUENTES PRIMARIAS}

Unicef. Convención Sobre los Derechos del Niño.

https: / / www.unicef.es / publicacion/convencion-sobre-los-derechosdel-nino,2006.

Uruguay. Ley No 17.823/004, de 7 de setiembre, de aprobación del Código de la Niñez y la Adolescencia. Dirección Nacional de Impresiones y Publicaciones Oficiales, 14 de setiembre de 2004, p.1

Uruguay. Ley $\mathrm{N}^{\circ}$ 19747. Modificaciones del Capítulo XI de la Ley 17.823, Código de la Niñez y la Adolescencia. Creación del SIPIAV. Dirección Nacional de Impresiones y Publicaciones Oficiales, 2019.

Fecha de recepción: 20 de agosto de 2021

Fecha de aceptación: 30 de noviembre de 2021 